\title{
ON THE SURVIVAL AND ABUNDANCE OF DISK-DOMINATED GALAXIES
}

\author{
Jun Koda ${ }^{1}$, Miloš Milosavluević ${ }^{2}$, And Paul R. Shapiro ${ }^{2}$ \\ ${ }^{1}$ Department of Physics, Texas Cosmology Center, University of Texas, 1 University Station C1600, Austin, TX 78712, USA \\ ${ }^{2}$ Department of Astronomy, Texas Cosmology Center, University of Texas, 1 University Station C1400, Austin, TX 78712, USA \\ Received 2007 November 19; accepted 2009 February 10; published 2009 April 14
}

\begin{abstract}
We study the formation of disk-dominated galaxies in a $\Lambda$ cold dark matter $(\Lambda \mathrm{CDM})$ universe. Their existence is considered to be a challenge for the $\Lambda \mathrm{CDM}$ cosmology, because galaxy mergers isotropize stellar disks and trigger angular momentum transport in gas disks, thus fostering the formation of central stellar spheroids. Here, we postulate that the formation of stellar spheroids from gas-rich disks is controlled by two parameters that characterize galaxy mergers, the mass ratio of merging dark matter halos, and the virial velocity of the larger merging halo. We utilize merger histories generated from realizations of the cosmological density field to calculate the fraction of dark matter halos that have avoided spheroid formation, and compare the derived statistics with the spheroid occupation fractions in surveys of nearby galaxies. We find, for example, that the survival rate of disk-dominated galaxies in $\Lambda \mathrm{CDM}$ is just high enough to explain the observed fractional representation of diskdominated galaxies in the universe if the only mergers which lead to central spheroid formation are those with mass ratios $M_{2} / M_{1}>0.3$ and virial velocities $V_{\mathrm{vir}, 1}>55 \mathrm{~km} \mathrm{~s}^{-1}$. We discuss the physical origin of this criterion.
\end{abstract}

Key words: cosmology: theory - galaxies: formation - galaxies: spiral

\section{INTRODUCTION}

The existence of disk-dominated galaxies, with little or no bulge, is frequently cited as a challenge to the $\Lambda$ cold dark matter $(\Lambda \mathrm{CDM})$ cosmology (e.g., Kautsch et al. 2006; Kormendy 2008). Apart from the question of whether or not the theory of galaxy formation in $\Lambda \mathrm{CDM}$ can succeed in making these observed rotationally-supported disk galaxies in the first place (D'Onghia \& Burkert 2004; Abadi et al. 2003), the survival of such disks, once formed, is our focus here. The mergers that every galaxy-hosting dark matter halo experiences can trigger angular momentum transport in the interstellar medium of the merger remnant. If a substantial amount of gas is transported into the central kiloparsec of the remnant, the gas can fuel a starburst forming a central stellar system-a "classical" bulge (i.e., self-gravitating, baryon-dominated stellar system supported by random motions rather than rotation). By contrast, "pseudobulges" can also arise in some disk galaxies if they have not experienced recent major mergers, by the secular transport of angular momentum (e.g., by galactic bars; Jogee et al. 2005). These pseudobulges (sometimes called "disky bulges") are supported more by rotation than random motion, however (e.g., Kormendy \& Kennicutt 2004 and references therein). ${ }^{3}$ About one third of all disk galaxies in the local universe do not contain bulges or pseudobulges (Kautsch et al. 2006) ${ }^{4}$ and another third contain only pseudobulges. Allen et al. (2006) carried out a Sérsic spheroid and exponential disk decomposition on a large sample of galaxies and find that $30 \%$ of exponential disks have small bulge-to-total ratio $B / T<0.2$. Barazza et al. (2008)

\footnotetext{
3 Pseudobulges are to a larger degree supported by rotation than classical bulges and can have rotational velocity-to-one-dimensional velocity dispersion ratios $V_{\max } / \sigma>1$. Their velocity dispersions are smaller than expected from the Faber-Jackson relation. Pseudobulges tend to have flattened isophotes and surface brightness profiles close to exponential. The classical bulges and pseudobulges can be distinguished from a third class, the boxy or peanut-shaped bulges, which are bars seen edge-on (Bureau \& Freeman 1999; Athanassoula 2005).

4 The classification of galaxies by their bulge content is usually done using simple bulge-disk or concentration models, which may not be accurate in extreme disk-dominated galaxies (e.g., Böker et al. 2003).
}

report that $20 \%$ of disk galaxies can be visually classified as bulgeless. ${ }^{5}$

For our purposes here, we shall assume that disk dominance means that no classical bulge is present in a galaxy. Although the precise characteristics of mergers that form classical bulges remain unknown, disk-dominated galaxies must have avoided major mergers during and after formation. Just how large the mass ratio of the merging galactic halos must be in order to induce bulge formation is somewhat uncertain, however. Mergers of similar-mass galaxies have been shown to trigger starbursts and result in elliptical galaxies, by gas dynamical and $N$-body simulations for galaxy halo masses $M \gtrsim 10^{11} M_{\odot}$, merging at relative velocities on the order of the virial velocity (Mihos \& Hernquist 1996; Cox et al. 2008). In that case, a bulge forms from the momentum-exchange and compression of gas in merger shocks and the outward angular momentum transport induced by merger torques. Pure $N$-body simulations of such mergers find that the pre-existing stellar disks are mixed and destroyed (e.g., Naab \& Burkert 2003), leaving an elliptical galaxy. For minor mergers, $N$-body and gas dynamical simulations with halo masses $M \gtrsim 10^{11} M_{\odot}$ indicate that disks survive but bulges can also grow (Mihos \& Hernquist 1994, Cox et al. 2008; Eliche-Moral et al. 2006, see also D'Onghia et al. 2006). However, pure $N$-body simulations of this process find that these bulges are pseudobulge-like (i.e., partially supported by rotation), rather than classical, for mass ratios $0.1 \lesssim M_{2} / M_{1} \lesssim 0.25$ (Bournaud et al. 2005), where $M_{1}$ and $M_{2}$ are the masses of the larger and smaller merging galaxy-hosting dark matter halos, respectively; hereafter, the subscripts 1 and 2 denote quantities pertaining to the larger and smaller halos, respectively. Bulge formation by the merging of lower mass $\left(V_{\max } \lesssim 70 \mathrm{~km} \mathrm{~s}^{-1}\right)$ and gas-rich galaxies has not yet been simulated.

\footnotetext{
5 Pure disk galaxies also contain nuclear star clusters (e.g., Böker et al. 2002; Walcher et al. 2005) which could be products of secular gas transport (Milosavljević 2004), but these star clusters are tiny by comparison with the bulges described above.
} 
Semianalytic models of galaxy formation (e.g., Kauffmann et al. 1993; Baugh et al. 1996) assume they can track the morphological type of galaxies by converting a disk component into a spheroidal component in mergers with mass ratios $\mu \equiv M_{2} / M_{1}$ greater than some threshold. These models successfully reproduce the distribution of earlier morphological types by tuning the critical mass ratio for disk destruction, and adopting a critical bulge-to-total mass ratio that discriminates broadly between disk and elliptical galaxies. Recent semianalytic models employing merger trees extracted from hydrodynamic $N$-body simulations yield a similar result (Maller et al. 2006). However, the studies that focus on the survival of disks generally do not address the abundance of disk-dominated galaxies. Existing ab initio cosmological simulations have yielded disks with bulges (e.g., Abadi et al. 2003), but currently lack the dynamic range to explore a large enough volume to sample the statistics of galaxy morphology while simultaneously resolving the formation and mergers of individual galaxies.

The purpose of this work is to compare the predicted disk survival probabilities during hierarchical merging in a $\Lambda \mathrm{CDM}$ universe with the observed statistics of galaxies at the end of the Hubble sequence. This comparison is then used to place constraints on the physics of bulge-forming mergers. In Section 2.1 we discuss the role of mergers in bulge formation. In Section 2.2 we describe the effect of cosmic reionization and Jeans-mass filtering on bulge formation. In Section 2.3 we present a calculation of bulge formation and disk survival probabilities derived from galactic halo merger trees generated from realizations of cosmological density fields. In Section 2.4 we compare observed disk galaxy statistics with these merger tree results and place constraints on the properties of bulge-forming mergers. In Section 3 we summarize our main conclusions. Standard cosmological parameters consistent with the Wilkinson Microwave Anisotropy Probe (Spergel et al. 2007) are assumed throughout.

\section{DISK SURVIVAL IN $\lambda$ CDM COSMOLOGY}

\subsection{Mechanisms for Bulge Formation in Mergers}

The merging of gas-rich galaxies fosters bulge formation directly and indirectly. Directly, the time-dependent gravitational potential of the two merging components deflects some of the gas into the center of the merger remnant, where it gets compressed in shocks and fuels a starburst. Indirectly, the gravitational tidal field of the merging components excites nonaxisymmetric perturbations inside the merging galaxies (bars, spirals, etc.) which then torque disk gas into the center of the galaxy (e.g., Combes 1998, and references therein). In the center, again, shocks are ubiquitous and play a role in angular momentum transport. The indirect channel should be important in minor mergers, especially where the smaller galaxy loses its gas to ram pressure stripping in the early stages of the merger.

The strength of direct merger torques is a function of the mass ratio of the host dark matter halos of the merging galaxies, $\mu \equiv M_{2} / M_{1}$. The strength of nonaxisymmetric distortions in minor mergers with $\mu \ll 1$ depends nontrivially on the resonance structure of the merging halos and the orbit of the merger (e.g., Goldreich \& Tremaine 1980). These cannot be modeled in a general case; therefore, we here consider only the gross properties of the mergers, averaged over the merger orbital parameters and over the properties of merging halos with given masses at a specific redshift.
Efficient transport of angular momentum in perturbed gas disks requires an excitation of nonlinear waves by nonaxisymmetric potential distortions that can give rise to momentumtransporting shocks. ${ }^{6}$ The strength of merger shocks is characterized by the Mach number $\mathcal{M}$, which is the ratio of the shock velocity to the sound speed of the warm neutral gas $c_{\mathrm{s}} \sim 10 \mathrm{~km} \mathrm{~s}^{-1}$. Merger-driven strong shocks can be radiative, because the post-shock cooling time can be much shorter than the dynamical and sound crossing times of the $\mathrm{H}$ I disk. In this limit the shocks are isothermal and the shock compression is $\sim \mathcal{M}^{2}$. This shock compression may in turn affect the rate of gravitational instability in the shocked gas and, hence, the efficiency of star formation.

In view of these considerations, we postulate that, besides the mass ratio $\mu$, the efficiency of bulge formation in mergers is also controlled by a second parameter, the merger Mach number $\mathcal{M} \equiv V_{\mathrm{vir}, 1} / c_{\mathrm{s}}$, where $V_{\mathrm{vir}, 1}$ is the virial velocity of the larger merging halo. ${ }^{7}$ In general, the condition that determines whether a merger triggers bulge formation or not can be any function of $\mu$ and $\mathcal{M}$. Here, we do not study the gas flow in merging galaxies, and hence we cannot specify the requirements for bulge formation in detail. Instead, we postulate a simple model of bulge formation that is characterized by two parameters, $\mu_{\text {crit }}$ and $\mathcal{M}_{\text {vir,crit }}$; bulges form if mergers with $\mu>\mu_{\text {crit }}$ and $\mathcal{M}>\mathcal{M}_{\text {vir,crit }}$ occur, but otherwise do not. By our definition of Mach number, the condition on a Mach number $\mathcal{M}>\mathcal{M}_{\text {crit }}$ is equivalent to a condition on the virial velocity $V_{\text {vir, } 1}>V_{\text {vir,crit }}=c_{s} \mathcal{M}_{\text {crit }}$, where $c_{s} \sim 10 \mathrm{~km} \mathrm{~s}^{-1}$ is the sound speed of gas.

An important distinct possibility is that in which bulge formation is driven cumulatively, rather than induced in a single merger (Bournaud et al. 2007). The central density of gas in the disk of a late-type disk galaxy could increase gradually due to slow, continuous radial gas inflow. Evidence for such inflow can be found in the "central light excess" (above the exponential law) in pure disk galaxies (e.g., Böker et al. 2003). The inflow could be excited by perturbations associated with minor mergers. Through their differential gravitational perturbations, many consecutive minor mergers can induce a slow, secular drift in the angular momentum distribution of the disk fluid, which could lead to central accumulation without leaving any characteristic signatures of merger-driven evolution (dynamically hot stellar components, etc.). The inflow could also be driven by nongravitational processes, such as the magnetorotational instability in the gas disk (Milosavljević 2004; see also, e.g., Piontek \& Ostriker 2004). The resulting increase of the central surface density brings the galaxy closer to the threshold for gravitational instability. The bulge or pseudobulge formation-triggering merger then must only nudge the galaxy over the threshold for, e.g., nuclear bar formation, where the galaxy is already marginally unstable.

For systems which are gas-poor, collisionless mergers of stars can result in an elliptical galaxy or a classical bulge, if and only if the $\mu$ is sufficiently large (e.g., Naab \& Burkert 2003; Bournaud et al. 2005). This bulge formation can be characterized by merger ratio $\mu$, but it is independent of $V_{\text {vir,1 }}$ because the gravitational dynamics without gas is scale free. However, there will still be a dependence on $V_{\mathrm{vir}, 1}$ through the requirement that the galaxies

\footnotetext{
6 For angular momentum transport by spiral shocks, see, e.g., Rozyczka \& Spruit (1993), Savonije et al. (1994), and Goodman \& Rafikov (2001). 7 Here and throughout the letter, $V_{\text {vir }} \equiv\left(G M_{200} / r_{200}\right)^{1 / 2}$, where $M_{200}$ is the mass inside a sphere with radius $r_{200}$ centered on the halo within which the mean density equals 200 times the critical density of the universe.
} 
prior to their merger were able to form long-lived (i.e., lowmass) stars (see below).

\subsection{The Critical Virial Velocity for Bulge Formation after Reionization and Jeans-Mass Filtering}

In order for the merger of two halos to have produced a bulge, the halos must have contained a substantial amount of gas, or else stars will have already formed from the collapsed gas. During cosmic reionization, in the process known as the Jeans-mass filtering (Shapiro et al. 1994), the gas content of small-mass halos, however, was affected by the reheating of the intergalactic medium (IGM) out of which the gas inside those halos collapsed. The gas pressure of the reheated intergalactic medium competes with gravitational instability, in that case, to suppress structure formation in those baryons which would otherwise have formed galaxies with virial velocity below some threshold. The Jeans length in the IGM for a gas photoheated to $\sim 10^{4} \mathrm{~K}$ corresponds to a halo mass after collapse and virialization for which the circular velocity is

$$
V_{\mathrm{c}}=55\left(T_{\mathrm{IGM}} / 10^{4} \mathrm{~K}\right)^{1 / 2} \mathrm{~km} \mathrm{~s}^{-1}
$$

(Iliev et al. 2007). The actual threshold virial velocity is uncertain, because one must account for the time-dependent growth of fluctuations in an evolving background and because the formation of dark matter halos affects the baryons in a nonlinear way. Estimates of the value of the velocity threshold which results range from about 30 to $80 \mathrm{~km} \mathrm{~s}^{-1}$ (e.g., Efstathiou 1992; Thoul \& Weinberg 1996; Navarro \& Steinmetz 1997; Kitayama \& Ikeuchi 2000). Whatever the precise value should be, this would impose a lower limit to the critical virial velocity of merging halos capable of producing a bulge, as described above, i.e., $V_{\text {crit, } \min } \sim 30-80 \mathrm{~km} \mathrm{~s}^{-1}$.

Since the virial velocity threshold which results from Jeansmass filtering depends primarily on the temperature, other sources of IGM heating could have a similar effect. The supernova explosions associated with massive star formation, for example, could also heat the intergalactic gas. Such feedback could also have unbound the interstellar gas from the galaxies which formed these stars, if the galaxy virial velocities were small enough.

\subsection{Merger Histories of Low-Mass Galaxies}

To explore the sensitivity of the fractional abundance of diskdominated galaxies produced during structure formation to the critical values of $\mu$ and $V_{\mathrm{vir}, 1}$, and thus to place constraints on the values of these two parameters that are compatible with the observed statistics, we generate merger histories of low-mass, disk-galaxy hosting halos and study disk survival in this population of halos. We utilize those merger histories to calculate the abundance of disk-dominated galaxies as a function of $\mu_{\text {crit }}$ and $V_{\text {vir,crit }}$. We compare the resulting abundances with the incidence of late-type galaxies in the Tully Galaxy Catalog (Section 2.4).

The merger histories are generated from the nonlinear evolution of the initial, linearly perturbed cosmological density field using the publicly-available Lagrangian perturbation code PINOCCHIO (Monaco et al. 2002). The code generates a Gaussian-random field on a cubic mesh, distributes particles on the mesh, and determines the collapse time of each particle using an ellipsoidal collapse criterion. The "collapsed" particles are moved by Lagrangian perturbation theory and related to virialized objects, which are the dark matter halos, by a linking criterion. We employed $512^{3}$ particles with cosmological parameters $\Omega_{\mathrm{m}}=0.24, \Omega_{\Lambda}=0.76, \sigma_{8}=0.74$, and $h=0.73$, in a cubical box with 50 comoving Mpc on a side. The mass of an individual particle was $m_{\text {part }}=3.3 \times 10^{7} M_{\odot}$, and halos with more than 10 particles were selected for inclusion in the merger tree. For a given redshift, PINOCCHIO provides a list of all of the halos with mass $M>10 m_{\text {part }}$ which formed inside the comoving box at this or any earlier redshift, and a complete list of their merger events. Each merger event is characterized by the merger redshift and the masses of the halos participating in the merger.

We compute the fraction of halos containing disk-dominated galaxies as a function of the threshold for spheroid formation that is parametrized by the critical mass ratio $\mu$ and critical virial velocity $V_{\mathrm{vir}, 1}$ of the larger halo at the time of each merger. Specifically, we assume that a merger with $\mu>\mu_{\text {crit }}$ will create a central stellar spheroid if the halo has a virial velocity $V_{\text {vir, } 1}>V_{\text {vir,crit }}$. We follow the most massive progenitor branch of the merger history of each halo and identify the resulting $z=0$ halo as containing a disk-dominated galaxy if no spheroid has yet formed in the halo based on the defined criterion.

There are rare cases in which a progenitor mass is so small at high redshift that bulge-forming mergers are not resolved by our numerical results. We have estimated the number of such cases and confirmed that it is negligible. For $1 / 10<\mu_{\text {crit }}<1 / 3$, the fraction of current halos in the mass range we will describe below, in which bulge-forming mergers occur with a halo containing less than 50 particles is less than $3 \%$. For $\mu_{\text {crit }}=1 / 2$, the fraction increases to $8 \%$, but the total mass of the merger remnant is small for those mergers with $\mu>1 / 2$. If the smaller halo contains 50 particles, the merger remnant with $\mu>1 / 2$ has at most 150 particles, which is ten times smaller than $M_{\min }$, the minimum mass of interest for our comparisons with present-day galaxies.

We consider halos with present masses in the range $M_{\min }<$ $M<M_{\max }$, where $M_{\min }=5 \times 10^{10} M_{\odot}$ (corresponding to $V_{\text {vir, } \min } \approx 60 \mathrm{~km} \mathrm{~s}^{-1}$ ) and $M_{\max }=10^{12} M_{\odot}$ (corresponding to $V_{\text {vir,max }} \approx 160 \mathrm{~km} \mathrm{~s}^{-1}$ ); the resulting galaxy statistics are compared with the observed galaxy statistics in the same approximate mass range. Since the halo mass and the maximum circular velocity of the galaxy disk are not known for most of the galaxies in each observed sample, we use the Tully-Fisher relation to estimate halo masses for the observed galaxies. We used the Tully-Fisher relation measured by Kannappan et al. (2002). The present total number density of halos in the above range is $0.021 \mathrm{Mpc}^{-3}$. Disk-dominated galaxy abundances thus calculated will not dependent strongly on the specific choice of $M_{\max }$ because halos with masses $M<M_{\max }$ dominate the number density of halos in the universe today. However, the abundances will be sensitive to $M_{\min }$. In Section 2.4 we explore the sensitivity to the choice of $M_{\min }$. An approach more accurate than the one employed here would dispense with $M_{\text {min }}$ and would consider halos of all masses and then match the fractional disk and irregular galaxy abundances as a function of halo mass.

We ignore the finite duration of the merger, which is the time elapsed between the halo contact and the final bulge formation. This approximation is generally good as long as the dynamical friction time for a galaxy to complete its merger with a central galaxy is shorter than the age of the universe when the halo merger took place. The dynamical friction time scale was 


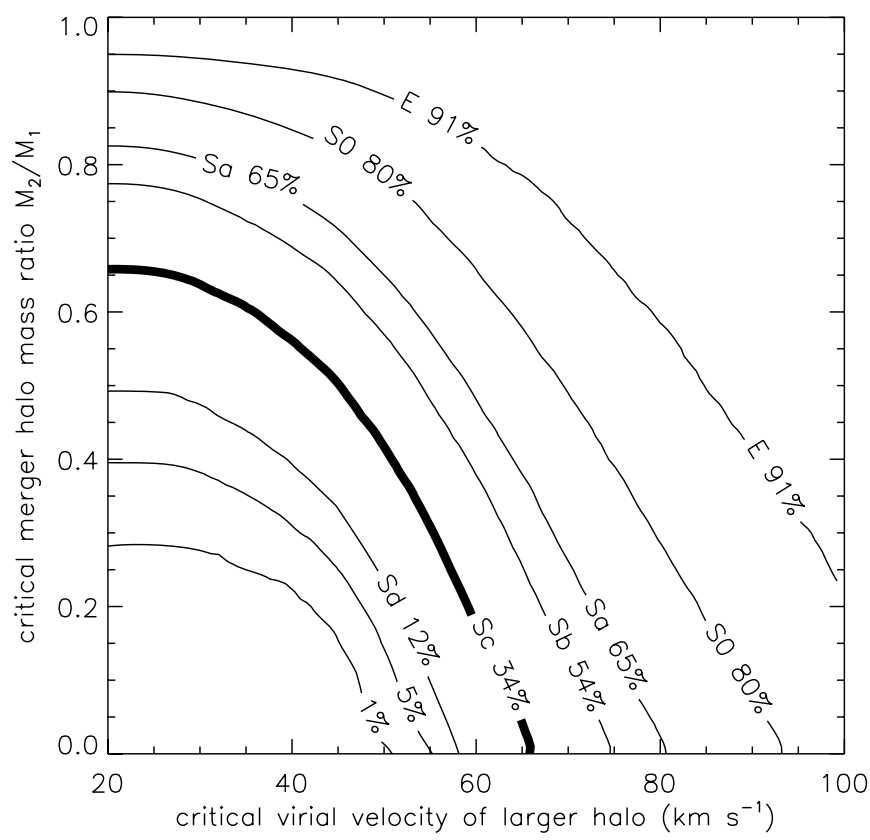

Figure 1. Fraction of disk-dominated galaxies (galaxies without classical bulges) that results from bulge formation criteria $\left(\mu_{\text {crit }}, V_{\text {vir,crit }}\right)$ characterized by the critical merger mass ratio, $\mu_{\text {crit }}$, and the critical virial velocity of the larger halo at merger, $V_{\text {vir,crit }}$. Some of the contours are also labeled by the morphological type of galaxy in the Tully subsample such that the observed fraction of galaxies of that morphological type or later equals the theoretical fraction of disk-dominated galaxies which results from the bulge-formation criterion $\left(\mu_{\text {crit }}, V_{\text {vir,crit }}\right)$ on that contour. As discussed in the text, we have adopted the view that galaxies of type Sc and later are the disk-dominated population (i.e., with no classical bulge), so the bold contour constitutes our theoretical expectation for the bulge-forming threshold criteria that is compatible with the observations.

recently calibrated in $N$-body simulations (Jiang et al. 2008),

$$
t_{\mathrm{dyn}}=\frac{0.94 \epsilon^{0.60}+0.60}{0.86} \frac{1}{\mu \ln \left(1+\mu^{-1}\right)} \frac{r_{\mathrm{vir}}}{V_{\mathrm{circ}}},
$$

where $\epsilon$ is the circularity parameter. Setting $\epsilon=0.5$ and $V_{\text {circ }}=V_{200}$, this simplifies to

$$
t_{\mathrm{dyn}} \sim \frac{0.14}{\mu} \frac{1}{\ln \left(1+\mu^{-1}\right)} H(z)^{-1},
$$

where $H(z)^{-1}$ is the Hubble time at halo merger. The dynamical friction time scale is smaller than the Hubble time for $\mu>0.05$, and therefore the galaxy merger time due to dynamical friction is negligible in most cases. Boylan-Kolchin et al. (2008) also gives a fitting formula for $t_{\mathrm{dyn}}$, which is different in detail (especially in its dependence on $\epsilon$ ), but also indicates that the dynamical friction time is smaller than the Hubble time for $\mu>0.05$ for any $\epsilon$. For late mergers, close to the present, we should perhaps compare this dynamical friction time with the look-back time to that merger event, rather than with the age of the universe then. The late mergers are only a minority, however. In the halo mass range corresponding to disk-dominated galaxy hosts, the present fraction of mergers in progress is only $\lesssim 5 \%$ for mass ratios $\mu>0.2$ and $\lesssim 10 \%$ for $\mu>0.1$.

\subsection{Results}

Figure 1 shows the fraction of galaxies without classical bulge as a function of the bulge formation criterion $\left(\mu_{\text {crit }}, V_{\mathrm{vir}, \text { crit }}\right)$, which is the result of the model described in Section 2.3. In order to compare these theoretical contours with the observed abundance of disk-dominated galaxies, we select 2281 galaxies in the nearby universe from the Tully Galaxy Catalog ${ }^{8}$ that have blue magnitudes in the range $-20<M_{B}<-17$ and are located at distances $D<20 h^{-1} \mathrm{Mpc}$ at which the catalog is reasonably complete. This luminosity range is chosen to render the number density of galaxies equal to the density $0.021 \mathrm{Mpc}^{-3}$ of halos that we synthesize (see Section 2.3). The corresponding circular velocity range calculated from the Tully-Fisher relation (e.g., Kannappan et al. 2002) is $60 \mathrm{~km} \mathrm{~s}^{-1}<V_{\mathrm{c}}<160 \mathrm{~km} \mathrm{~s}^{-1}$. This range is consistent with the range of virial velocities in our theoretical halo sample, which is a self-consistency check of our association of galaxies with dark matter halos in our numerical halo catalog for $\Lambda \mathrm{CDM}$.

Some of the contours in Figure 1 are labeled with a galaxy morphological type, as well. Each pair of morphological type and fraction printed on the contour indicates that that morphological type and later types occupy the corresponding fraction in the subsample of the Tully catalog. If we choose to assume that a particular morphological type (e.g., Sd, Sc, or Sb) and all later morphological types are disk-dominated galaxies, while the earlier morphological types are galaxies with classical bulges, then the fraction of disk-dominated galaxies in the subsample is explained by the parameters $\left(\mu_{\text {crit }}, V_{\text {vir,crit }}\right)$ along the contour labeled by the chosen transitional morphological type. The classification by morphological type does not precisely separate galaxies that have classical bulges from those do not; nevertheless we assume that $\mathrm{Sc}$ and later type galaxies are either bulgeless or have pseudobulges, while Sbc and earlier types contain classical bulges. Assuming this correspondence, the contour "Sc" in the figure shows the parameter space locus yielding the abundance of galaxies without classical bulges.

While the criterion for the survival in mergers of a given morphological type must lie on the appropriate contour, from the statistics alone it cannot be determined which specific value of $\left(\mu_{\text {crit }}, V_{\text {vir,crit }}\right)$ along the contour is the true physical criterion for bulge formation. The critical virial velocity cannot be much less than the minimum value imposed by Jeans-mass filtering discussed in Section 2.2. If $V_{\text {vir,crit }}=V_{\text {crit,min }}=55 \mathrm{~km} \mathrm{~s}^{-1}$ (see Equation (1)), then $\mu_{\text {crit }}$ is 0.3 on the Sc contour. That is, the observed abundance of disk-dominated galaxies is consistent with the hypothesis that mergers with $\mu>0.3$ in halos with $V_{\text {vir, } 1}>55 \mathrm{~km} \mathrm{~s}^{-1}$ create classical bulges, while mergers that do not satisfy these criteria do not. This $\mu_{\text {crit }}$ is consistent with the threshold value such that mergers with larger mass ratio result in a random-motion-dominated ellipsoid in dissipationless $N$-body simulations (Bournaud et al. 2005). However, since the precise value of $V_{\text {vir }}$, below which gas collapse is suppressed is not known, a somewhat larger $\mu_{\text {crit }}$ and somewhat smaller $V_{\text {vir,crit }}$ and vice versa would be equally plausible, e.g., $\left(\mu_{\text {cirt }}, V_{\text {vir,cirt }}\right)=$ $\left(0.3_{-0.2}^{+0.3}, 55_{+5}^{-10} \mathrm{~km} \mathrm{~s}^{-1}\right)$.

Were the critical velocity for bulge formation above $\sim 65 \mathrm{~km} \mathrm{~s}^{-1}$, however, then the relative abundance of diskdominated galaxies would be greater than observed for all mass ratios $\mu$. In that case, there would be too few galaxies with bulges relative to their observed abundance. A similar upper limit to $V_{\text {vir,crit }}$ results from the fact that classical bulges are unlikely to form from mergers that are too minor. If $V_{\mathrm{vir}, \text { crit }} \sim 65 \mathrm{~km} \mathrm{~s}^{-1}$, then mergers will over-produce disk-dominated galaxies unless $\mu_{\text {crit }} \ll 1$, which may be implausibly small (i.e., not major mergers). To identify the true, unique criterion for bulge formation, one must resort to physical insight to exclude

\footnotetext{
8 http://haydenplanetarium.org/universe/duguide/exgg_tully.php.
} 


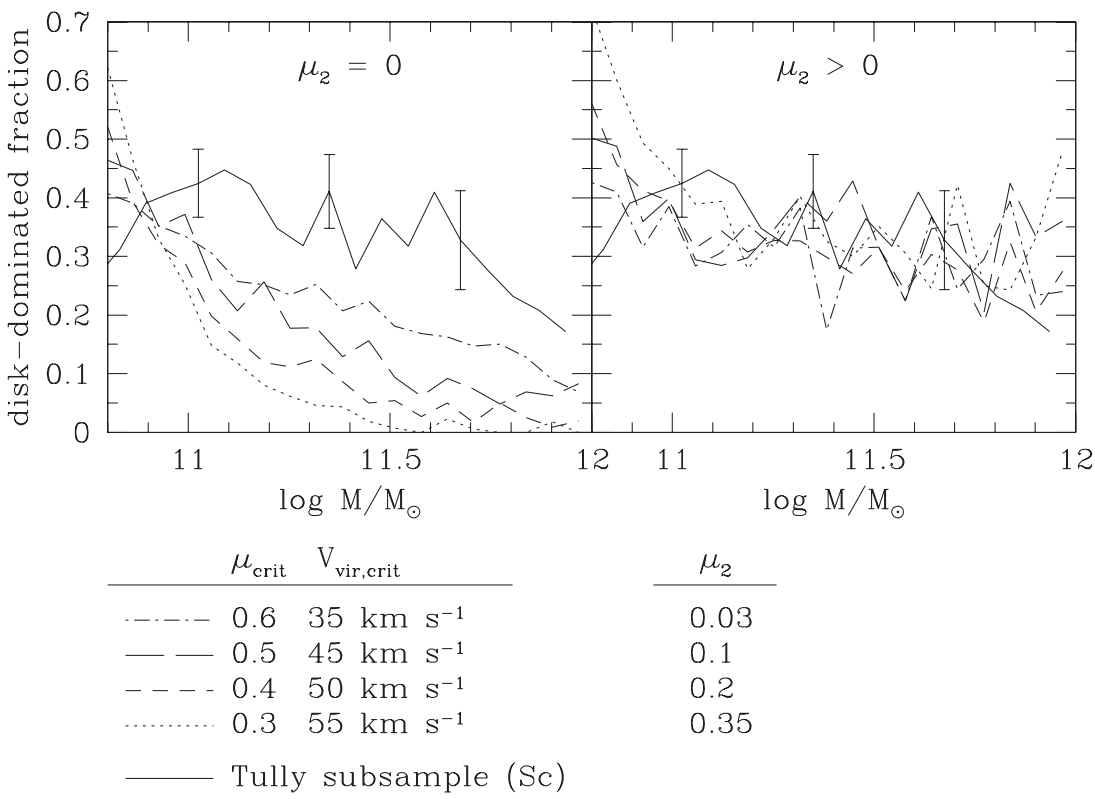

Figure 2. Disk-dominated fraction as a function of galaxy-hosting halo Mass for the two-parameter model ( $\left.\mu_{\text {crit }}, V_{\text {vir,crit }}\right)$ (left panel) and the three-parameter model with additional parameter $\mu_{2}$ (right panel), in which we assume that a merger remnant would not be observed as a classical bulge if its mass were smaller than $\mu_{2}$ times present halo mass. Solid line is the observed fraction of Sc and later in the Tully subsample with Poisson error bars. Our model with two parameters does not agree with the observed mass dependence, but the agreement become reasonably good by introducing the third parameter $\mu_{2}$.

such implausible, extreme criteria that are still allowed by the statistics.

The Kautsch et al. (2006) sample of edge-on disk galaxies gives a similar disk-dominated galaxy fraction. If we assume the same additional fraction of early-type galaxies as in the Tully subsample, the fraction of $\mathrm{Sd}$ is $11 \%$ and the fraction of Sc or later is $45 \%$.

The assumption that the Tully-Fisher relation can be used to relate the luminosities of galaxies in the Tully catalog to their halo virial velocities (and, hence, to their masses, $M_{200}$ ) works best for the spiral galaxies but is less certain for the elliptical and $\mathrm{S} 0$ galaxies. The Tully-Fisher relation for S0 galaxies is shifted to lower luminosity by about $M_{B} \sim+1.5$ for each $V_{\max }$, and the scatter is larger compared to spirals (Bedregal et al. 2006). This estimate is uncertain because the Tully-Fisher relation or virial mass-to-light ratio is unknown for S0s at small masses near $M_{\min }$. The virial mass-to-light ratio for elliptical galaxies could be a factor of 10 larger relative to spirals in the $B$-band (Hoekstra et al. 2005; Guzik \& Seljak 2002). If we shift the luminosity range of the subsample that corresponds to $M_{\min }<M<M_{\max }$ by $+5 \mathrm{mag}$ for ellipticals and $+1.5 \mathrm{mag}$ for S0s, respectively, then all the ellipticals will be removed (i.e., $M>M_{\max }$ ) and the number of S0s will increase by 30\%, but the sum of E and S0 will only decrease from $25 \%$ to $20 \%$, and the Sc contour move from $34 \%$ to $36 \%$. Hence, uncertainties regarding the virial velocities of the ellipticals and S0s in the sample do not significantly affect our determination of the disk-dominated portion.

To test the dependence on mass cutoff $M_{\min }$, we vary this parameter from the fiducial cutoff at $5 \times 10^{10} M_{\odot}$ to a lower cutoff at $3.2 \times 10^{10} M_{\odot}$. The number density of halos increases by $50 \%$ to $0.034 \mathrm{Mpc}^{-3}$. To compensate for the change in the number density of galaxies, we move the lower luminosity cutoff for selection from the Tully sample to $M_{B}<-15.5$. The observed fraction of disk-dominated galaxies ( $\mathrm{Sc}$ or later morphological type) remains unchanged at $\approx 33 \%$, but the contours representing the bulge formation criterion move by about $10 \%$ toward lower critical virial velocities. This is because mergers with a fixed mass ratio $\mu$ tend to occur at smaller
$V_{\text {vir, } 1}$ in smaller halos. The criterion, e.g., with $\left(\mu_{\text {crit }}, V_{\text {vir,crit }}\right)=$ $\left(\frac{1}{4}, 57 \mathrm{~km} \mathrm{~s}^{-1}\right)$ on the Sc contour in Figure 1 shifts only by a small amount, to $\left(\frac{1}{4}, 50 \mathrm{~km} \mathrm{~s}^{-1}\right)$, as the mass cutoff is lowered to $3.2 \times 10^{10} M_{\odot}$. Any lower cutoffs than this are not appropriate, because at luminosities $M_{B} \lesssim-15$, disk galaxies give way to irregulars as the most common galaxy type (e.g., Binggeli et al. 1988).

In Figure 1, we showed that the integrated fraction of diskdominated galaxies in the mass range $M_{\min }<M<M_{\max }$ can be explained by a two-parameter model with $\left(\mu_{\text {crit }}, V_{\text {vir,crit }}\right)$ along the Sc contour in that figure. However, we can also compare the disk-dominated fraction in our model with observations as a function of halo mass. The left panel of Figure 2 shows the diskdominated fraction as a function of mass for the Tully subsample (Sc or later) and our model with $\left(\mu_{\text {crit }}, V_{\text {vir,crit }}\right)=(0.3,55)$, $(0.4,50),(0.5,45)$, and $(0.6,35)$, respectively, which are points along the Sc contour in Figure 1. Our model predicts that diskdominated fractions decrease too quickly with mass, compared to observation. The quick decline is understandable, because halos rarely accrete mass from $V_{\text {vir }}=V_{\text {vir,crit }}$ to $M \sim 10^{11.5}$ $10^{12} M_{\odot}$ without major merger, i.e., $\mu>\mu_{\text {crit }}$. However, these classical bulges formed when $V_{\text {vir }} \sim V_{\text {vir,crit }}$ may be too small to be observed for such large halos $M \sim 10^{11.5}-10^{12} M_{\odot}$. To show this effect, we add a third criterion with a free parameter $\mu_{2}$, which determines that the classical bulge is large enough to be observed only if

$$
\left(M_{1}+M_{2}\right)\left(z_{\mathrm{mer}}\right)>\mu_{2} M_{0}
$$

where $z_{\text {mer }}$ is a redshift of a merger, and $M_{0}$ is the halo mass at present. With this additional condition, the diskdominated fraction is consistent with the Tully subsample for, e.g., $\left(\mu_{\text {crit }}, V_{\text {vir,crit }}, \mu_{2}\right)=(0.3,55,0.35),(0.4,50,0.2)$, $(0.5,45,0.1)$, or $(0.6,35,0.03)$. To make a better prediction, one must track the disk and bulge mass separately along the merger tree, and predict the bulge-to-total ratio, as often done in semianalytic galaxy formation models, which is beyond the scope of this paper. 


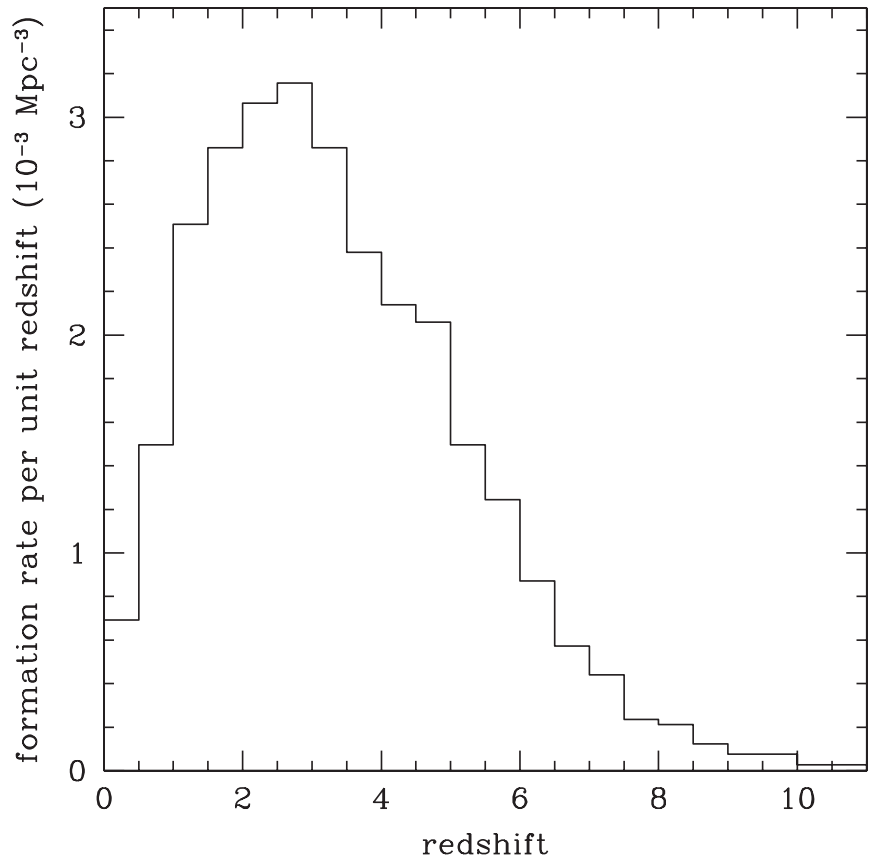

Figure 3. Redshift distribution of first mergers that create classical bulges. We assumed $\mu_{\text {crit }}=0.3$ and $V_{\text {vir,crit }}=55 \mathrm{~km} \mathrm{~s}^{-1}$ as the critical parameters for parameters of bulge formation, which is consistent with the abundance of disk-dominated galaxies in the local universe (see Figure 1).

In Figure 3, we plot the redshift distribution of the earliest bulge-forming mergers of $z=0$ halos for $\mu_{\text {crit }}=0.3$ and $V_{\text {vir,crit }}=55 \mathrm{~km} \mathrm{~s}^{-1}$. 9 The distribution is insensitive to the choices of $\mu_{\text {crit }}$ and $V_{\text {vir,crit }}$, as long as the two parameters remain on the same contour in Figure 1. This shows that bulgeforming mergers generally took place long after reionization was completed (i.e., $z_{\text {rei }} \gtrsim 6$ ). Moreover, the typical collapse epoch for these merging halos was at $z<6$ (Shapiro \& Iliev 2002), so their star formation was also post-reionization. Hence the lower limit to $V_{\text {vir,crit }}$ imposed by Jeans-mass filtering is applicable.

Minor mergers may not destroy galactic disks, but they can heat and thicken disks. Toth \& Ostriker (1992) placed an upper limit on the amount of merging that could have taken place during the lifetime of a galactic disk by quantifying the role of mergers in the heating and thickening of the Milky Way's disk. They found that in order for the Milky Way disk to have remained thin, the Galaxy could not have accreted more than $5 \%$ of its present mass during the past 5 Gyr. Subsequent work refined the estimates of disk heating, resulting in less stringent constraints on the merger history (e.g., Velazquez \& White 1999; Benson et al. 2004). An $N$-body simulation of mergers shows that a thin disk survives if $\mu<0.02$ (Kazantzidis et al. 2008).

Kauffmann \& White (1993) used the Press-Schechter excursion set theory to generate merger histories of Milky Way sized halos in a matter-dominated CDM universe (i.e., $\Lambda=0$ ) and addressed the Toth-Ostriker constraint by calculating the fraction of halos that experienced a merger with mass ratio larger than $\mu$ after redshift $z$. Their results suggested that, while the Toth and Ostriker constraint might be difficult to satisfy in an Einstein-de

\footnotetext{
9 If, as commonly assumed, bulge-forming mergers are responsible for quasar activity, it would be temping to point out the resemblance between the redshift distribution of first bulge-forming mergers and the observed evolution of the quasar luminosity (Richards et al. 2006). However, the mass range of the halos in Figure 3 is significantly lower than that of the halos thought to host bright quasars.
}

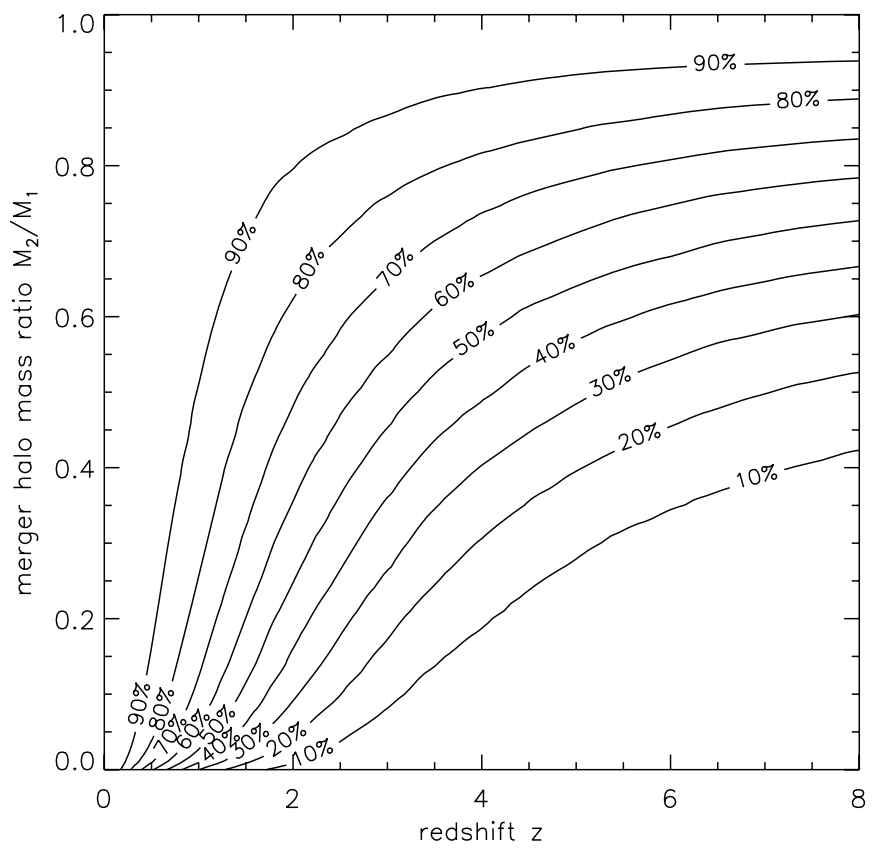

Figure 4. Fraction of halos that have not experienced a merger with mass ratio $M_{2} / M_{1}$ or larger since redshift $z$.

Sitter universe, an open universe had no such difficulty. We have used our merger history calculation to recompute this statistic for the currently favored $\Lambda \mathrm{CDM}$ universe, as shown in Figure 4 . We find that, since $z=1,70 \%$ of the halos throughout the mass range from $5 \times 10^{10} M_{\odot}$ to $10^{12} M_{\odot}$ had no merger with $\mu>0.05$. This fraction is larger than that reported by Kauffmann \& White (1993) for their open universe case. This suggests that thin-disks survive the destructive effects of merger identified by Toth \& Ostriker (1992).

Although the Toth \& Ostriker constraint is probably satisfied in $\Lambda \mathrm{CDM}$, the time period $5 \mathrm{Gyr}$ is only a lower bound for the age of the disk. It is not certain weather thin disks survive during the whole history of galaxies. Stewart et al. (2008) assume the age of thin disks to be $\sim 10 \mathrm{Gyr}$ instead, consistent with estimates for the Milky Way. They conclude that whenever subhalos as large as $\sim 2 \times 10^{11}$ merge with Milky Way sized galaxies anytime during the last $10 \mathrm{Gyr}$, their thin disks must survive, or else the $70 \%$ fraction of disk galaxies cannot be explained, but such survival seems unlikely. However, the typical present-day Milky Way-mass galaxies assembled most of their mass more recently then $\sim 10$ Gyr ago, so it may not be necessary to require that typical thin-disks survived that long. Recently, Hopkins et al. (2008) have claimed that mergers along nearly radial orbits (common in $\Lambda \mathrm{CDM}$ ) heat the disk less than Toth-Ostriker's estimate because the infalling galaxy is rapidly tidally stripped. They said that even several 1:10 mergers could be consistent with the survival of the Milky Way thin disk.

\section{CONCLUSIONS}

In order to explain the observed space density and fraction of disk-dominated galaxies within the $\Lambda \mathrm{CDM}$ cosmology, we propose a bulge-forming criterion such that only those halo mergers with mass ratio greater than $\mu_{\text {crit }} \sim 0.3$ and halo virial velocity (of the larger halo) above $V_{\text {vir,crit }} \sim 55 \mathrm{~km} \mathrm{~s}^{-1}$ formed classical bulges, while other mergers did not. This criterion has some degeneracy between $\mu_{\text {crit }}$ and $V_{\text {vir,crit }}$, but $V_{\text {vir,crit }}$ cannot be larger than about $65 \mathrm{~km} \mathrm{~s}^{-1}$ without underproducing the 
galaxy fraction with bulges, or much smaller than $\sim 60 \mathrm{~km} \mathrm{~s}^{-1}$ since Jeans-mass filtering after reionization inhibits such smallmass halos from acquiring and retaining baryons or forming stars. The validity of this bulge formation criterion needs to be confirmed by further analytic calculation or hydrodynamical simulations of major and minor mergers in the halo mass range $V_{\text {vir, } 1} \lesssim 60 \mathrm{~km} \mathrm{~s}^{-1}$.

After we submitted this paper, Hopkins et al. (2009) analyzed galaxy-merger simulations for a wide range of merger parameters and presented an analytic model that predicts the post-merger disk fraction as a function of galaxy and merger properties. They found that the bulge dominated fraction among the merger remnants depends strongly on the gas fraction of the galaxies; with a larger gas fraction, bulge formation is less efficient. This is qualitatively consistent with our suggestion that bulge do not form for $V_{\text {vir }}<V_{\text {vir,crit }}$, because galaxies near the Jeans-mass filtering scale should be gas rich. Their simulations adopted a range of assumed gas fractions for the merging galaxies, but they did not attempt to determine the values of this gas fraction self-consistently for the $\Lambda \mathrm{CDM}$ universe. So we cannot directly compare the simulation results with our statistical analysis. Their results can, in principle, be incorporated into semi-analytic galaxy formation models, which estimate the gas fractions of merging galaxies, to improve the prediction of the disk-dominated fraction and other morphological properties in $\Lambda \mathrm{CDM}$.

We find that bulge-forming criteria in our model that can explain the observed total disk-dominated galaxy fraction tend to predict that mass-dependent disk-dominated fraction decreases more rapidly with increasing halo mass than is observed. However, the discrepancy disappears if the bulges formed by mergers that involved halos with masses much smaller than the final halo mass are difficult to detect. Future surveys of bulgeless/pseudobulged galaxy fraction as a function of mass should be a useful discriminant of models such as ours and improve our knowledge of galaxy and bulge formation.

We thank Shardha Jogee for detailed comments, and John Kormendy for inspiring and illuminating discussions. We thank the anonymous referee for helpful comments. This work was supported in part by NSF grant AST-0708795 to M. M., and NASA ATP grants NNG04G177G, NNX07AH09G, SAO TM89009X, and NSF grant AST-0708176 to P. R. S.

\section{REFERENCES}

Abadi, M. G., Navarro, J. F., Steinmetz, M., \& Eke, V. R. 2003, ApJ, 591, 499

Allen, P. D., Driver, S. P., Graham, A. W., Cameron, E., Liske, J., \& de Propris, R. 2006, MNRAS, 371, 2

Athanassoula, E. 2005, MNRAS, 358, 1477

Barazza, F. D., Jogee, S., \& Marinova, I. 2008, ApJ, 675, 1194

Baugh, C. M., Cole, S., \& Frenk, C. S. 1996, MNRAS, 283, 1361

Bedregal, A. G., Aragón-Salamanca, A., \& Merrifield, M. R. 2006, MNRAS, 373,1125

Benson, A. J., Lacey, C. G., Frenk, C. S., Baugh, C. M., \& Cole, S. 2004, MNRAS, 351, 1215
Binggeli, B., Sandage, A., \& Tammann, G. A. 1988, ARA\&A, 26, 509

Böker, T., Laine, S., van der Marel, R. P., Sarzi, M., Rix, H.-W., Ho, L. C., \& Shields, J. C. 2002, AJ, 123, 1389

Böker, T., Stanek, R., \& van der Marel, R. P. 2003, AJ, 125, 1073

Bournaud, F., Jog, C. J., \& Combes, F. 2005, A\&A, 437, 69

Bournaud, F., Jog, C. J., \& Combes, F. 2007, A\&A, 476, 1179

Bureau, M., \& Freeman, K. C. 1999, AJ, 118, 126

Boylan-Kolchin, M., Ma, C.-P., \& Quataert, E. 2008, MNRAS, 383, 93

Combes, F. 1998, in Starbursts: Triggers, Nature, and Evolution, Les Houches School, ed. B. Guiderdoni \& A. Kembhavi (Berlin: Springer), 175

Cox, T. J., Jonsson, P., Somerville, R. S., Primack, J. R., \& Dekel, A. 2008, MNRAS, 384, 386

D'Onghia, E., \& Burkert, A. 2004, ApJ, 612, L13

D'Onghia, E., Burkert, A., Murante, G., \& Khochfar, S. 2006, MNRAS, 372, 1525

Efstathiou, G. 1992, MNRAS, 256, 43P

Goodman, J., \& Rafikov, R. R. 2001, ApJ, 552, 793

Goldreich, P., \& Tremaine, S. 1980, ApJ, 241, 425

Guzik, J., \& Seljak, U. 2002, MNRAS, 335, 311

Eliche-Moral, M. C., Balcells, M., Aguerri, J. A. L., \& González-García, A. C. 2006, A\&A, 457, 91

Hoekstra, H., Hsieh, B. C., Yee, H. K. C., Lin, H., \& Gladders, M. D. 2005, ApJ, 635,73

Hopkins, P. F., Cox, T. J., Younger, J. D., \& Hernquist, L. 2009, ApJ, 691, 1168

Hopkins, P. F., Hernquist, L., Cox, T. J., Younger, J. D., \& Besla, G. 2008, ApJ, 688,757

Iliev, I. T., Mellema, G., Shapiro, P. R., \& Pen, U.-L. 2007, MNRAS, 376, 534

Jiang, C. Y., Jing, Y. P., Faltenbacher, A., Lin, W. P., \& Li, C. 2008, ApJ, 675, 1095

Jogee, S., Scoville, N., \& Kenney, J. D. P. 2005, ApJ, 630, 837

Kannappan, S. J., Fabricant, D. G., \& Franx, M. 2002, AJ, 123, 2358

Kauffmann, G., \& White, S. D. M. 1993, MNRAS, 261, 921

Kauffmann, G., White, S. D. M., \& Guiderdoni, B. 1993, MNRAS, 264, 201

Kautsch, S. J., Grebel, E. K., Barazza, F. D., \& Gallagher, J. S., III. 2006, A\&A, 445,765

Kazantzidis, S., Bullock, J. S., Zentner, A. R., Kravtsov, A. V., \& Moustakas, L. A. 2008, ApJ, 688, 254

Kormendy, J., \& Kennicutt, R. C., Jr. 2004, ARA\&A, 42, 603

Kormendy, J. 2008, in IAU Symp. 245, Formation and Evolution of Galaxy Bulges, ed. M. Bureau, E. Athanassoula, \& B. Barbuy (Cambridge: Cambridge Univ. Press), 107

Kitayama, T., \& Ikeuchi, S. 2000, ApJ, 529, 615

Maller, A. H., Katz, N., Kereš, D., Davé, R., \& Weinberg, D. H. 2006, ApJ, 647, 763

Mihos, J. C., \& Hernquist, L. 1994, ApJ, 425, L13

Mihos, J. C., \& Hernquist, L. 1996, ApJ, 464, 641

Milosavljević, M. 2004, ApJ, 605, L13

Monaco, P., Theuns, T., \& Taffoni, G. 2002, MNRAS, 331, 587

Naab, T., \& Burkert, A. 2003, ApJ, 597, 893

Navarro, J. F., Frenk, C. S., \& White, S. D. M. 1997, ApJ, 490, 493

Navarro, J. F., \& Steinmetz, M. 1997, ApJ, 478, 13

Piontek, R. A., \& Ostriker, E. C. 2004, ApJ, 601, 905

Richards, G. T., et al. 2006, AJ, 131, 2766

Rozyczka, M., \& Spruit, H. C. 1993, ApJ, 417, 677

Savonije, G. J., Papaloizou, J. C. B., \& Lin, D. N. C. 1994, MNRAS, 268, 13

Shapiro, P. R., Giroux, M. L., \& Babul, A. 1994, ApJ, 427, 25

Shapiro, P. R., \& Iliev, I. T. 2002, ApJ, 565, L1

Spergel, D. N., et al. 2007, ApJS, 170, 377

Stewart, K. R., Bullock, J. S., Wechsler, R. H., Maller, A. H., \& Zentner, A. R. 2008, ApJ, 683, 597

Thoul, A. A., \& Weinberg, D. H. 1996, ApJ, 465, 608

Toth, G., \& Ostriker, J. P. 1992, ApJ, 389, 5

Velazquez, H., \& White, S. D. M. 1999, MNRAS, 304, 254

Walcher, C. J., et al. 2005, ApJ, 618, 237 